\title{
FIXED POINTS FOR PAIRS OF MAPPINGS IN $d$-COMPLETE TOPOLOGICAL SPACES
}

TROY L. HICKS

\author{
Department of Mathematics \\ University of Missouri - Rolla \\ Rolla, MO 65401 U.S.A. \\ and
}

\section{B.E. RHOADES}

Department of Mathematics Indiana University Bloomington, IN 47405 U.S.A.

(Received October 7, 1991 and in revised form March 12, 1992)

\begin{abstract}
Several important metric space fixed point theorems are proved for a large class of non-metric spaces. In some cases the metric space proofs need only minor changes. This is surprising since the distance function used need not be symmetric and need not satisfy the triangular inequality.
\end{abstract}

KEY WORDS AND PHRASES. Commuting, compatible, $d$-complete topological spaces, fixed points, pairs of mappings.

1991 SUBJECT CLASSIFICATION CODE. 47H10, 54H25.

\section{INTRODUCTION.}

Let $(X, t)$ be a topological space and $d: X \times X \rightarrow[0, \infty)$ such that $d(x, y)=0$ if and only if $x=y . \quad X$ is said to be $d$-complete if $\sum_{n=1}^{\infty} d\left(x_{n}, x_{n+1}\right)<\infty$ implies that the sequence is convergent in $(X, t)$. In a metric space, such a sequence is a Cauchy sequence. $T: X \rightarrow X$ is $w$-continuous at $x$, if $x_{n} \rightarrow x$ implies $T x_{n} \rightarrow T x$.

Definition. A symmetric on a set $X$ is a real-valued function $d$ on $X \times X$ such that:

(1) $d(x, y) \geq 0$ and $d(x, y)=0$ if and only if $x=y$; and

(2) $d(x, y)=d(y, x)$.

Let $d$ be a symmetric on a set $X$ and for any $\epsilon>0$ and any $x \in X$, let $S(x, \epsilon)=\{y \in X$ : $d(x, y)<\epsilon\}$. We define a topology $t(d)$ on $X$ by $U \in t(d)$ if and only if for each $x \in U$, some $S(x, \epsilon) \subseteq U$. A symmetric $d$ is a semi-metric if for each $x \in X$ and each $\epsilon>0, S(x, \epsilon)$ is a neighborhood of $x$ in the topology $t(d)$. A topological space $X$ is said to be symmetrizable (semi-netrizable) if its topology is induced by a symmetric (semi-metric) on $X$.

The $d$-complete synmetrizable (semi-metrizable) spaces form an important class of examples of $d$-complete topological spaces. We give other examples. Let $X$ be an infinite set and $t$ any $T_{1}$ non-discrete first countable topology for $X$. Then there exists a complete nuctric 
I for $X$ such that $t \leq t_{d}$ and the metric topology $t_{d}$ is non-discrete. Now $(X, t)$ is $d$-complete since $\sum d\left(x_{n}, x_{n+1}\right)<\infty$ implies that $\left\{x_{n}\right\}$ is a $d$-Cauchy sequence. Thus, $x_{n} \rightarrow x$ in $t_{d}$ and therefore in the topology $t$. See [4] for the construction of $t_{d}$. It should also be noted that any complete quasi-metric space $(X, d) \quad(d(x, y) \neq d(y, x))$ is a $d$-complete topological space. These are several competing definitions for a Cauchy sequence, but $\sum d\left(x_{n}, x_{n+1}\right)<\infty$ will inply that $\left\{x_{n}\right\}$ is a Cauchy sequence for any reasonable definition. One reasonable definition is derived from requiring that the filter generated by $\left\{x_{n}\right\}$ be a Cauchy filter in the quasiunifornity generated by $d$. This gives $\left\{x_{n}\right\}$ is a Cauchy sequence if for each $\epsilon>0$ there exists a positive integer $n_{0}=n(\epsilon)$ and $x=x(\epsilon)$ in $X$ such that $\left\{x_{n}: n \geq x_{0}\right\} \subseteq\{y \in X: d(x, y)<\epsilon\}$. The metric space definition also holds if $\sum d\left(x_{n}, x_{n+1}\right)<\infty$. Some fundamental theorems for d-complete topological spaces are given in [4] and [5].

2. RESULTS. The results through Corollary 2.2 are generalizations of theorems due to Jungck [i]. Even though only minor changes are needed in the proofs, they are given for completeness.

LEMMA 2.1. Let $(X, t)$ be a d-complete topological space. If there exists $\alpha \in(0,1)$ such that $d\left(y_{n}, y_{n+1}\right) \leq \alpha d\left(y_{n-1}, y_{n}\right)$ for all $n$, then $\left\{y_{n}\right\}$ converges to a point in $X$.

PROOF. $d\left(y_{n}, y_{n+1}\right) \leq \alpha^{n-1} d\left(y_{1}, y_{2}\right)$ gives $\sum_{n=1}^{\infty} d\left(y_{n}, y_{n+1}\right) \leq d\left(y_{1}, y_{2}\right) \sum_{n=1}^{\infty} \alpha^{n-1}<\infty$. The result follows since $X$ is $d$-complete.

THEOREM 2.1. Let $(X, t)$ be a Hausdorff $d$-complete topological space and $f: X \rightarrow X$ a (w) continuous function. Then $f$ has a fixed point in $X$ if and only if there exists $\alpha \in(0,1)$ and a u-continuous function $g: X \rightarrow X$ which commutes with $f$ and satisifes

(1) $g\left(X^{-}\right) \subseteq f(X)$ and $d(g x, g y) \leq \alpha d(f x, f y)$ for all $x, y \in X$. Indeed, $f$ and $g$ have a unique common fixed point if (1) holds.

PROOF. Suppose $f(a)=a$ for some $a \in X$. Put $g(x)=a$ for all $x \in X$. Then $g(f x)=a$ and $f(g, x)=f(a)=a$. Also, $g(x)=a=f(a)$ for all $x \in X$ so that $g(X) \subseteq f(X)$. For any a $\in(0,1), d(g x, g y)=d(a, a)=0 \leq \alpha d(f x, f y)$. Since $g$ is $w$-continuous, (1) holds.

Suppose there exists $\alpha$ and $g$ such that (1) holds. Let $x_{0} \in X$ and let $x_{n}$ be such that (2) $f\left(x_{n}\right)=g\left(x_{n-1}\right)$. Now (1) and (2) gives $d\left(f x_{n}, f x_{n+1}\right)=d\left(g x_{n-1}, g x_{n}\right) \leq \alpha d\left(f x_{n-1}, f x_{n}\right)$. Lemma 2.1 gives $p$ in $X$ such that $f\left(x_{n}\right) \rightarrow p . g$-continuous implies that $\lim g f x_{n}=g p$. Since $g x_{n} \rightarrow p$, the $w$-continuity of $f$ implies that $\lim f g x_{n} \rightarrow f p$. Therefore $p$ is a coincidence point of $f$ and $g$. Clearly, $f g p=g f p$.

$f(f p)=f(g p)=g(f p)=g(g p)$ and $d(g p, g(g p)) \leq \alpha d(f p, f(g p))=\alpha d(g p, g(g p))$. Hence, $g(p)=g(g p)$. Thus $g(p)=g(g p)=f(g p)$, and $g(p)$ is a common fixed point of $f$ and $g$.

If $x=f(x)=g(x)$ and $y=f(y)=g(y)$, then (1) gives $d(x, y)=d(g x, g y) \leq \alpha d(f x, f y)=$ $\alpha d(x, y)$ or $x=y$.

COROLLARY 2.1. Let $(X, t)$ be a Hausdorff $d$-complete topological space and $f$ and $g$ commuting mappings from $X$ to $X$. Suppose $f$ and $g$ are $w$-continuous and $g(X) \subseteq f(X)$. If there exists $\alpha \in(0,1)$ and a positive integer $k$ such that

$$
d\left(g^{k} x, g^{k} y\right) \leq \alpha d(f x, f y) \text { for all } x, y \in X,
$$


then $f$ and $g$ have a unique common fixed point.

PROOF. Clearly, $g^{k}$ commutes with $f$ and $g^{k}(X) \subseteq g(X) \subseteq f(X)$. Applying the theorem to $g^{k}$ and $f$ gives a unique $p \in X$ with $p=g^{k}(p)=f(p)$. Since $f$ and $g$ commutc $g(p)=$ $y(f p)=f(g(p))=g^{k}(g p)$ or $g(p)$ is a common fixed point of $f$ and $g^{k}$. Uniqueness gives $g(p)=p=f(p)$.

Letting $f=i$, the identity, gives a generalization of Banach's theorem. If we also let $k=1$, we get Banach's theorem in this new setting.

COROLLARY 2.2. Let $n$ be a positive integer and let $k>1$. If $g$ is a surjective ucontinuous self map of a Hausdorff d-complete topological space $X$ such that

$$
d\left(g^{n} x, g^{n} y\right) \geq k d(x, y) \text { for all } x, y \in X,
$$

then $g$ has a unique fixed point.

PROOF. Let $f=g^{2 n}$ and $\alpha=\frac{1}{k}$ in Corollary 2.1. Now $d(x, y) \leq \alpha d\left(g^{n} x, g^{n} y\right)$ gives $d\left(g^{n} x, g^{n} y\right) \leq \alpha d\left(g^{2 n} x, g^{2 n} y\right)=\alpha d(f x, f y) . \quad g$ surjective implies $g(X) \subseteq f\left(X^{*}\right)$.

THEOREM 2.2 Let $(X, t)$ be a Hausdorff d-complete topological space. Suppose $f$ and $g$ are commuting $\omega$-continuous selfmaps of $X$ such that $g(X) \subseteq f(X)$ and

(a) $d\left(g^{n} x, g^{n} y\right) \leq d_{n} d(f x, f y)$

for each $n$ and for all $x, y$ in $X$, where $d_{n}>0$ and $\sum_{n=1}^{\infty} d_{n}<\infty$.

Then:

(1) $f$ and $g$ have a unique common fixed point $p$.

(2) If $x \in X, \lim g^{n} x=\bar{x}$ exists, with $g \bar{x}=\bar{x}$.

(3) $f \bar{x}, f^{2} \bar{x}, \ldots$ are fixed points of $g$. If $p \neq \bar{x}$, then $p \notin\left\{f \bar{x}, f^{2} \bar{x}, \ldots,\right\}$. If $f \bar{x} \neq f^{2} \bar{x}$ then $f^{n} \bar{x} \neq f^{n+1} \bar{x}$ for each $n \geq 1$.

(4) If $\lim f^{n} \bar{x}=y$ exists, then $y=p$.

(5) If the topology $t$ is given by a metric $d$ then

$$
d\left(g^{n} x, \bar{x}\right) \leq\left(\sum_{k=n}^{\infty} d_{k}\right) d(f x, g f x) .
$$

If, in addition, $f$ is the identity, then $\bar{x}=p$ for each $x$ in $X$.

PROOF. Since $\lim d_{n}=0$, there exists $a k$ with $d_{k}<1$. Then

$$
d\left(g^{k} x, g^{k} y\right) \leq d_{k} d(f x, f y)
$$

From Corollary 2.1, $f$ and $g$ have a unique common fixed point $p$.

To prove (2), fix $x$ and set $y=g x$ in (a). Then $d\left(g^{n} x, g^{n+1} x\right) \leq d_{n} d(f x, f g x)$, which gives

$$
\sum_{n=1}^{\infty} d\left(g^{n} x, g^{n+1} x\right) \leq d(f x, f g x) \sum_{n=1}^{\infty} d(f x, g f x)<\infty .
$$

$X$ is $d$-complete, so there exists an $\bar{x}$ such that $x_{n}=g^{n} x \rightarrow \bar{x}$. Since $g$ is $\omega$-continuous $x_{n+1}=g\left(x_{n}\right) \rightarrow g \bar{x}$. Clearly $g \bar{x}=\bar{x}$. 
To prove (3), $g\left(f^{k} \bar{x}\right)=f^{k}(g \bar{x})=f^{k} \bar{x}$ for each $k \geq 1$. If $p \neq \bar{x}$ thien $0<d(p, \bar{x})=$ $d(g p, g \bar{x}) \leq d_{1} d(f p, f \bar{x})=d_{1} d(p, f \bar{x})$, and $p \neq f \bar{x}$. Thus $0<d(p, f \bar{x})=d(g p, g f . x) \leq$ $d_{1} d\left(f p, f^{2} \bar{x}\right)=d_{1} d\left(p, f^{2} \bar{x}\right)$ and $p \neq f^{2} \bar{x}$. By induction $p \neq f^{n} \bar{x}$ for any $n \geq 1$.

If $f \bar{x} \neq f^{2} \bar{x}$ then $0<d\left(f \bar{x}, f^{2} \bar{x}\right)=d\left(g f \bar{x}, g f^{2} \bar{x}\right) \leq d_{1} d\left(f^{2} \bar{x}, f^{3} \bar{x}\right)$ and $f^{2} \bar{x} \neq f^{3} \bar{x}$. By induction $f^{n} \bar{x} \neq f^{n+1}$, for cach $u \geq 1$.

To prove (4), $g f^{n} \bar{x} \rightarrow g y$ and $g f^{n} \bar{x}=f^{n} g \bar{x}=f^{n} \bar{x} \rightarrow y$ and $g y=y . \quad f^{n+1} \bar{x} \rightarrow y$ and $f^{n+1} \bar{x}=f f^{n} \ddot{x} \rightarrow f y$, so $f y=y$. From (1) $y=p$.

To prove (5),

$$
\begin{aligned}
d\left(g^{n} x, g^{n+r} x\right) & \leq \sum_{k=n}^{n+r-1} d\left(g^{k} x, g^{k+n} x\right) \leq \sum_{k=n}^{n+r-1} d_{k} d(f x, f g x) \\
& \leq d(f x, g f x) \sum_{k=n}^{\infty} d_{k} .
\end{aligned}
$$

Tilking the limit as $r \rightarrow \infty$ yields

$$
d\left(g^{n} x, \bar{x}\right) \leq d(f x, g f x) \sum_{k=n}^{\infty} d_{x} .
$$

If $f$ is the identity, $g$ has a unique fixed point from (1). But (2) gives $g \bar{x}=\bar{x}$. Therefore $\bar{x}=p$.

Altman [2] called $f: X \rightarrow X$ a generalized contraction provided $d(f x, f y) \leq Q(d(x, y))$ for all $x, y \in X$ where $Q$ satisfies the following:
(a) $0<Q(t)<t$ for all $t \in\left(0, t_{1}\right]$,
(b) $g(t)=t /(t-Q(t))$ is non-increasing,
(c) $\int_{0}^{t_{1}} g(t) d t<\infty$, and
(d) $Q$ is non-decreasing.

He assumes $(X, d)$ is a metric space and proves that for any $x_{0} \in X, x_{n}=f^{n} x_{0} \rightarrow x=f(x)$. We use his definition in the more general setting to obtain the following.

THEOREM 2.3. Let $(X, t)$ be a d-complete Hausdorff topological space and $f: X \rightarrow X$ a $w$-continuous generalized contraction. Let $x_{0} \in X$ and put $x_{n}=f^{n} x_{0}=f\left(x_{n-1}\right)$. Then $\lim x_{n}=x$ and $x=f(x)$.

PROOF. Put $s_{1}=d\left(x_{0}, x_{1}\right)$ and $s_{n+1}=Q\left(s_{n}\right)$ for $n \geq 1$. Using (a), (b), and (c), Altman shows that the series $\sum_{n=1}^{\infty} s_{n}$ generated by $Q$ is convergent. By induction,

$$
d\left(x_{n}, x_{n+1}\right)=d\left(f x_{n-1}, f x_{n}\right) \leq Q\left(d\left(x_{n-1}, x_{n}\right)\right) \leq Q\left(s_{n}\right)=s_{n+1} .
$$

Thus, $\sum_{n+1}^{\infty} d\left(x_{n}, x_{n+1}\right)<\infty$ and $\lim x_{n}=x$ exists. $f w$-continuous gives $x_{n+1}=f\left(x_{n}\right) \rightarrow$ $f(x)$. Thus, $f(x)=x$.

If one replaces topological by symmetrizable in the theorem, $f$ is forced to be $w$-continuous. Even though Altman asserted uniqueness of the fixed point, examples exist [11] that show otherwise.

Our next result is the $d$-complete analogue of Theorem 2.1 of Park [9].

THEOREM 2.4. Let $g$ and $h$ be selfmaps of a $d$-complete topological space $X$. if 


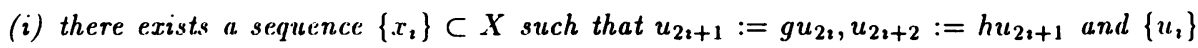
has a cluster point $\xi$ in $\mathrm{X}$,

(ii) $g$ and hg are $\omega$-continuous at $\xi$,

(izz) $G(x):=d(x, g x), H(x):=d(x, h x)$ are orbitally continuous at $\xi$, and

(iv) $g$ and $h$ satisfy

$$
d(g x, h y)<d(x, y)
$$

for each distinct $x$ and $y$ in $\left\{\overline{u_{2}}\right\}$ satisfying either $x=h y$ or $y=g x$, then

(1) g or h has a fixed point in $\left\{\overline{u_{2}}\right\}$ or

(2) $\xi$ is a common fixed point of $g$ and $h$ and $u_{2} \rightarrow \xi$ as $i \rightarrow \infty$.

The proof is the same as that of Theorem 2.1 of [9].

COROLLARY 2.3. Let $g$ and $h$ be $w$-continuous selfmaps of a d-complete space $X$ satisfying

$$
d(h x, g y) \leq k \max \{d(x, y), d(x, h x), d(y, g y), d(y, h x)\}
$$

for all $x, y$ in $X$, where $0 \leq k<1$ and $d(x, g x)$ and $d(x, h x)$ are orbitally continuous. Then $g$ or $h$ has a fixed point or $g$ and $h$ have a unique common fixed point.

PROOF. Let $u_{2 n+1}=h u_{2 n}, u_{2 n+2}=g u_{2 n+1}$. Thus, from the contractive definition,

$$
\begin{aligned}
d\left(u_{2 n+1}, u_{2 n+2}\right) \leq k \max \{ & d\left(u_{2 n}, u_{2 n+1}\right), d\left(u_{2 n}, u_{2 n+1}\right) \\
& \left.d\left(u_{2 n+1}, u_{2 n+2}\right), d\left(u_{2 n+1}, u_{2 n+1}\right)\right\}
\end{aligned}
$$

which implies that $d\left(u_{2 n+1}, u_{2 n+2}\right) \leq k d\left(u_{2 n}, u_{2 n+1}\right)$. Therefore $\left\{u_{n}\right\}$ is Cauchy, hence converges to a point $\xi$. The hypotheses of Theorem 4 are now satisfied.

The uniqueness of a common fixed point follows from the contractive definition.

We now demonstrate that several results in the literature follow as special cases of Theorem 2.4. While our list is not exhaustive, it indicates the generality of the theorem.

COROLLARY 2.4. [3, Theorem 1]. Let $T_{1}, T_{2}$ be two selfmaps of a Hausdorff $F$-complete space $X, F: X \times X \rightarrow[0, \infty)$ a continuous symmetric mapping such that $F(x, y)=0$ for $x=y$ and, for each pair of distinct $x, y$ in $X$,

$$
F\left(T_{1} x, T_{2} y\right)<\max \left\{\left[F(x, y), F\left(x, T_{1} x\right), F\left(y, T_{2} y\right)\right] \cup \min \left[F\left(y, T_{2} y\right), F\left(y, T_{1} x\right)\right]\right\}
$$

and for some $x_{0}$ in $X$ the sequence $\left\{x_{n}\right\}$ defined by $x_{2 n+1}=T_{1} x_{2 n}, x_{2 n+2}=T_{2} x_{2 n+1}$, has a subsequence converging to a point $\xi$ in $X$. If $T_{1}$ and $T_{2} T_{1}$ or $T_{2}$ and $T_{1} T_{2}$ are continuous at $\xi$, then $\xi$ is a fixed point of $T_{1}$ and $T_{2}$ (or $T_{1}$ or $T_{2}$ has a fixed point).

PROOF.

$$
\begin{gathered}
F\left(T_{1} x, T_{2} T_{1} x\right)<\max \left\{\left[F\left(x, T_{1} x\right), F\left(x, T_{1} x\right), F\left(T_{1} x, T_{2} T_{1} x\right)\right] \cup\right. \\
\min \left[F\left(T_{1} x, T_{2} T_{1} x\right), F\left(T_{1} x, T_{1} x\right)\right\} \\
=\max \left\{F\left(x, T_{1} x\right), F\left(T_{1} x, T_{2} T_{1} x\right)\right\}
\end{gathered}
$$

which implies that $F\left(T_{1} x, T_{2} T_{1} x\right)<F\left(x, T_{1} x\right)$, and condition (iv) of Theorem 2.3 is satisfied.

With $g=T_{1}, h=T_{2}$, the remaining conditions of Theorem 2.4 are satisfied. 
If we now set $g=T_{2}, h=T_{1}$ then, from the contractive definition,

$$
\begin{gathered}
F\left(T_{1} T_{2} x, T_{2} x\right)<\max \left\{\left[F\left(T_{2} x, x\right), F\left(T_{2} x, T_{1} T_{2} x\right), F\left(x, T_{2} x\right)\right]\right. \\
\left.\cup \min \left[F\left(x, T_{2} x\right), F\left(x, T_{1} T_{2} x\right)\right]\right\} \\
=\max \left\{F\left(T_{2} x, T_{1} T_{2} x\right), F\left(x, T_{2} x\right)\right\},
\end{gathered}
$$

which implies that $F\left(T_{1} T_{2} x, T_{2} x\right)<F\left(x, T_{2} x\right)$, and again the hypotheses of Theorem 2.4 are satisfied.

COROLLARY 2.5. [8, Theorem 1]. Let $f$ and $g$ be $\omega$-continuous selfmaps of a d-complete L-space $X$, with $d(x, x)=0$ and $d(x, y)=d(y, x)$ for each $x, y$ in $X$. If $f$ and $g$ satisfy

$$
\begin{gathered}
\min \left\{d^{2}(f x, g y), d(x, y) d(f x, g y), d^{2}(y, g y)\right\} \\
-\min \{d(x, f x) d(y, g y), d(x, g y) d(y, f x)\} \leq q d(x, f x) d(y, g y)
\end{gathered}
$$

for all $x$, in $X$, and $0<q<1$, then $f$ and $g$ have a common fixed point, (or $f$ or $g$ has a fixed. point).

PROOF. As in [8], it can be shown that $\left\{x_{n}\right\}$ converges, where $\left\{x_{n}\right\}$ is defined by $x_{2 n+1}=$ $f x_{2 n}, x_{2 n+2}=g x_{2 n+1}$.

Setting $y=f x$ in the contractive definition yields

$$
\begin{gathered}
\min \left\{d^{2}(f x, g f x), d(x, f x) d(f x, g f x), d^{2}(f x, g f x)\right\} \\
-\min \{d(x, f x) d(f x, g f x), d(x, g f x) d(f x, f x)\} \leq q d(x, f x) d(f x, g f x),
\end{gathered}
$$

or

$$
\min \left\{d^{2}(f x, g f x), d(x, f x) d(f x, g f x)\right\} \leq q d(x, f x) d(f x, g f x)
$$

If the minimum is $d^{2}(f x, g f x)$, then we have

$$
d^{2}(f x, g f x) \leq q d(x, f x) d(f x, g f x),
$$

which implies that either $f x$ is a fixed point of $g$, or $d(f x, g f x) \leq q d \dot{d}(x, f x)$.

If the minimum is $d(x, f x) d(f x, g f x)$, either $x$ is a fixed point of $f, f x$ is a fixed point of $g$, or $d(f x, g f x) \leq q d(x, f x)$. Therefore the conditions of Theorem 2.4 are satisfied, except for (iii).

However, for this contractive definition (iii) is not needed. As in [8], $\left\{x_{n}\right\}$ converges to a point $u$ in $X$. Since $f$ is $\omega$-continuous and $x_{2 n+1}=f x_{2 n}$, it follows that $u=f u$. Similarly, $g u=u$.

COROLLARY 2.6. [1, Theorem 1] Let $S, T$ be orbitally $\omega$-continuous selfmaps of a $d$ complete $L$-space $X$, with $d(x, y)=0$ iff $x=y$. If for some $0<\alpha<1$ and for each $x, y$ in $\mathbf{X}$,

$$
d(S x, T y) \leq \alpha\{d(x, S x) d(y, T y)\}^{1 / 2}
$$

and $d(x, S x), d(x, T x)$ are orbitally continuous, then $S$ and $T$ have a unique common fixed point (or $S$ or $T$ has a fixed point). 
PROOF. Note that the contractive definition implies that

$$
\begin{aligned}
d(S . x, T y) & \leq \alpha\left\{\max \left[d^{2}(x, S x), d^{2}(y, T y)\right]\right\}^{1 / 2} \\
& =\alpha \max \{d(x, S x), d(y, T y)\}
\end{aligned}
$$

and the result follows from Corollary 2.3.

COROLLARY 2.7. [10, Theorem 1]. Let $X$ be an $F$-complete Hausdorff space, $T_{1}, T_{2}$ continuous selfmaps of $X$. Let $F: X \times X \rightarrow \mathbb{R}_{+}, F$ continuous and such that $F(x, x)=0$ for all $x$ in $X$ and

$$
F\left(T_{1}^{p} x, T_{2}^{q} y\right)<a_{1} F(x, y)+a_{2} F\left(x, T_{1}^{p} x\right)+a_{3} F\left(y, T_{2}^{q} y\right)
$$

for each distinct $x, y$ in $X$, where $p$ and $q$ are positive integers, $a_{2} \geq 0, a_{1}+a_{2}+a_{3}<1$. If for some $x_{0} \in X$, the sequence $\left\{x_{n}\right\} \subset X$ defined by $x_{2 n+1}=T_{1}^{p} x_{2 n}, x_{2 n+2}=T_{2}^{q} x_{2 n+1}$ has a convergent subsequence, then ( $T_{1}$ or $T_{2}$ has a fixed point) or $T_{1}$ and $T_{2}$ have a unique common fixed point.

PROOF. Set $y=T_{1}^{p} x$ to get

$$
F\left(T_{1}^{p} x, T_{2}^{q} T_{1}^{p} x\right)<a_{1} F\left(x, T_{1}^{p} x\right)+a_{2} F\left(x, T_{1}^{p} x\right)+a_{3} F\left(T_{1}^{p} x, T_{2}^{q} T_{1}^{p} x\right),
$$

or

$$
F\left(T_{1}^{p} x, T_{2}^{q} T_{1}^{p} x\right)<\frac{a_{1}+a_{2}}{1-a_{3}} F\left(x, T_{1}^{p} x\right),
$$

and the conditions of Theorem 2.4 are satisfied with $f=T_{1}^{p}, g=T_{2}^{q}$.

The uniqueness follows as in [10].

REMARK. The words in parentheses in Corollaries 4-7 have been added by the present authors, in order for the theorems to be correctly stated. 


\section{REFERENCES}

1. B. Ahmad and Fazal-ur-Rehman, Fixed point theorems in $L$-spaces. Math. Japonica 36 (1991), 225-228.

2. M. Altman, An integral test for series and generalized contractions. Amer. Math. Monthly 82 (1975), 827-829.

3. H. Chatterjee and Barada K. Ray, On further extension of a theorem of Edelstein. Indlian J. Pure Appl. Math. 11 (1980), 256-257.

4. Troy L. Hicks and Wilson R. Crisler, A note on $T_{1}$ topologies. Proc. Amer. Math. Soc. 46, (1974), 94-96.

5. Troy L. Hicks, Fixed point theorems for $d$-complete topological spaces I. Int. J. Math. and Math. Sci., to appear.

6. Troy L. Hicks and B.E. Rhoades, Fixed point theorems for $d$-complete topological spaces II. Math. Japonica, to appear.

7. Gerald Jungck, Compatible mappings and common fixed points. Int. J. Math. and Math. Sci. 9 (1986), 771-779.

8. B.G. Pachpatte, Common fixed point theorems for certain mappings in $L$-spaces. J. M.A.C.T. 13 (1980), 55-58.

9. Sehie Park, Fixed points and periodic points of contractive pairs of maps. Proc. College of Nat. Sci., Seoul National Univ. 5 (1980), 9-22.

10. Barada K. Ray and H. Chatterjee, Some results on fixed points in metric and Banach spaces. Bull. L'Acad. Pol. des Sci. 25 (1977), 1243-1247.

11. B. Watson, B.A. Meade and C.W. Norris, Note on a theorem of Altman. Indian J. Pure Appl. Math. 17 (1986) 1092-1093. 


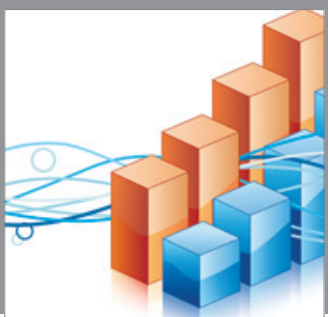

Advances in

Operations Research

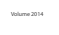

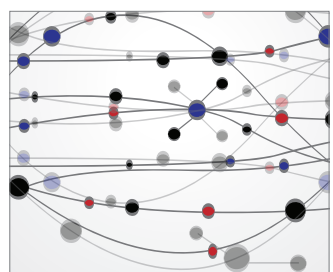

\section{The Scientific} World Journal
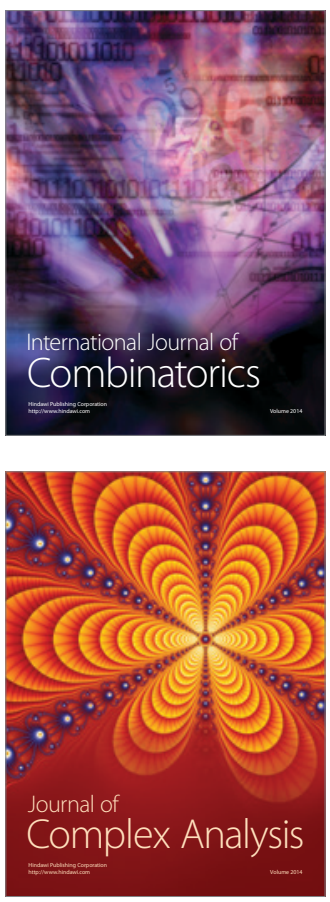

International Journal of

Mathematics and

Mathematical

Sciences
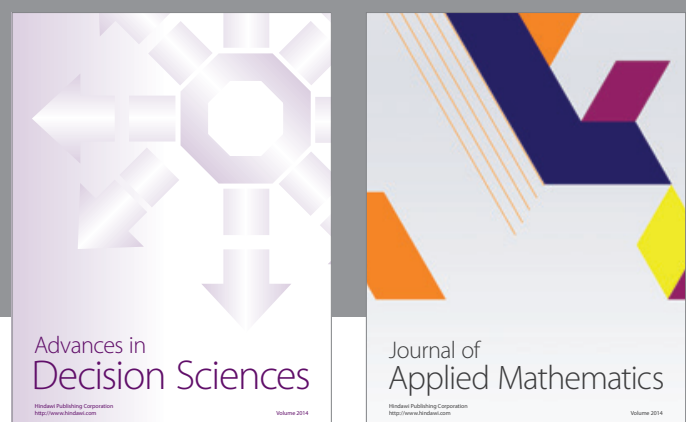

Journal of

Applied Mathematics
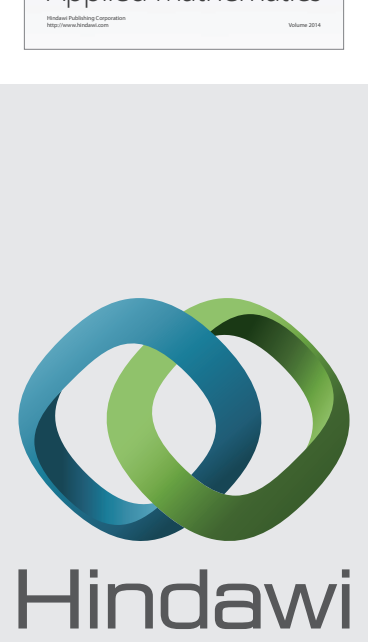

Submit your manuscripts at http://www.hindawi.com
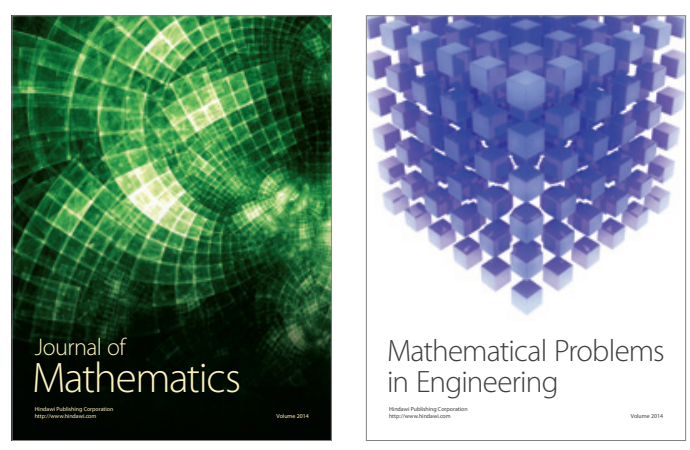

Mathematical Problems in Engineering
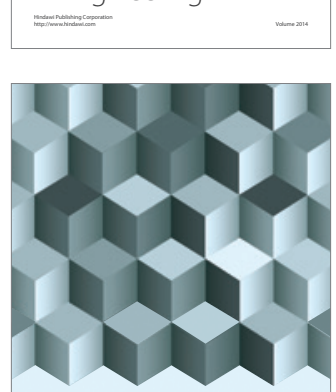

Journal of

Function Spaces
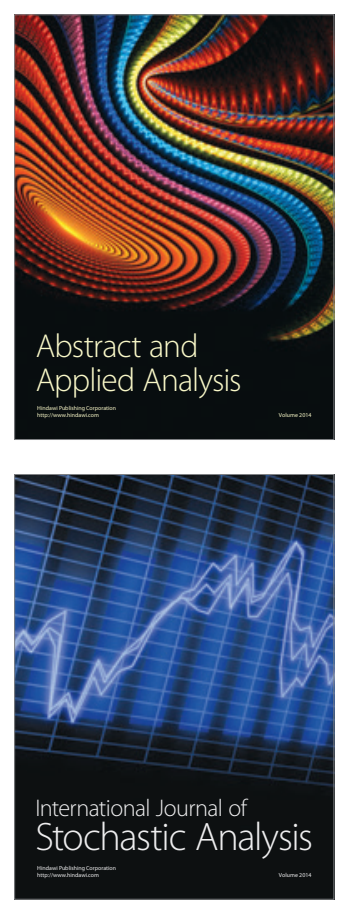

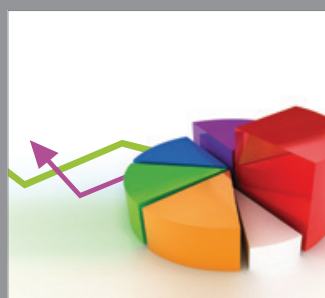

ournal of

Probability and Statistics

Promensencen
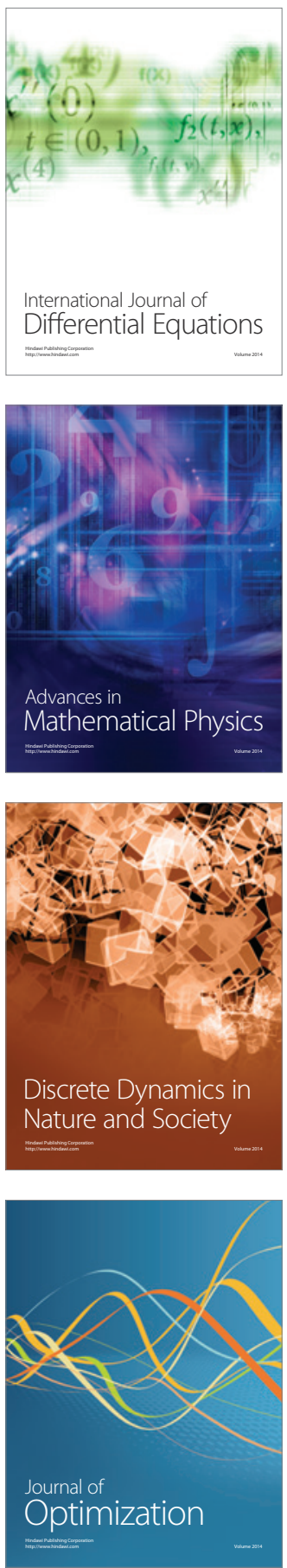